\title{
MEJORA DEL SISTEMA DE ALIMENTACIÓN EN LAS MÁQUINAS ENVASADORAS MULTIPACK Y ROVEMA PARA REDUCIR EL RIESGO ERGONOMICO Y LOS TIEMPOS IMPRODUCTIVOS DE OPERACIÓN EN EMPRESAS MANUFACTURERAS DE CHOCOLATES
}

\section{IMPROVEMENT OF THE SUPPLY SYSTEM IN MULTIPACK AND ROVEMA PACKAGING MACHINES TO REDUCE ERGONOMIC RISK AND IMPROPER OPERATING TIMES IN A MANUFACTURING COMPANY}

\author{
Ing. Martha Catalina Ospina Hernández*, Ing. Julián Andrés Angulo Arroyo* \\ PhD. Albert Miyer Suarez Castrillón ${ }^{* *}$, PhD. Sir Alexci Suarez Castrillón *** \\ * Universidad Nacional Abierta y a Distancia - UNAD, Escuela de Ciencias Básicas \\ Tecnología e Ingeniería. La Paila, Valle del Cauca, Colombia. \\ E-mail: \{anacamila41, ulianrro\}@ @otmail.com \\ ${ }^{* *}$ Universidad de Pamplona, Investigador Grupo GIMUP. \\ Km 1 via bucaramanga, Pamplona, Norte de Santander, Colombia. \\ $(57+7) 5685303$. \\ E-mail: albertmiyer@hotmail.com \\ **: Universidad Francisco de Paula Santander Ocaña, Investigador Grupo GRUCITE. \\ Vía Acolsure, Sede El Algodonal, Ocaña, Norte de Santander, Colombia. \\ $(57+7) 5690088$. \\ E-mail: sasuarezc@ufpso.edu.co
}

Resumen: En la ejecución de las actividades para alimentar las máquinas, se presenta una carga laboral excesiva, esto es debido a que se levantan manualmente las conchas cargadas de producto. Cada una de estas conchas tienen un peso entre 17.5 y 19.5 kilos, este peso es considerado viable bajo los estándares establecidos en la seguridad y salud del trabajador siempre y cuando se realicen en las condiciones y posturas correctas, pero las actividades específicas del proceso y el traslado de estas conchas son muy frecuentes, de esta forma se está realizando un esfuerzo ergonómico no adecuado. Debido al problema ergonómico se percibe la necesidad de implementar un sistema de alimentación para las máquinas aprovechando un elevador en " $Z$ " ya existente que pertenece a una máquina Robat.

Palabras clave: Multipack, Rovema, chocolates, riesgo ergonómico

\begin{abstract}
Packaging process is done by automatic machines Multipack y Rovema. In the activities to feed of product to the machines or lines, we always present a very high and heavy labour of work, due to all is done by hand and man power. Boxes full of filling product are lifted to get the Top of it, to be able to fill the product running through line. These boxes are always between 17 and $19 \mathrm{~kg}$; this Kind of weight is allowed by regulations of security in labours of work and regulations for behavior of all workers in plant. To all three lines, beginning in the highest part of the "Z" lifting system and ends in the rest of the Multipack, Rovema and Robat lifters.
\end{abstract}

Keywords: Multipack, Rovema, Chocolates, ergonomic risk 


\section{INTRODUCCIÓN}

En toda organización manufacturera o industrial, una de las áreas fundamentales resulta ser el área productiva, esta es donde se desarrollan los procesos que se enfocan a la satisfacción del cliente. Al hacer referencia al área de producción, es importante tener en cuenta al personal que realice sus actividades de una forma segura para su salud y bienestar (Chinchilla, 2005).

El trabajo se enfoca desde tres aspectos fundamentales: el Técnico, que afronta las actividades en el proceso siendo más eficientes; el Epistemológico, que permite el grado de conocimiento por el equipo de investigación y el innovador siendo la técnica fundamental a realizar en la investigación para el cambio y las mejoras del proceso. A través del desarrollo de esta investigación se busca entonces el diseño de puestos de trabajo adaptados a las características fisiológicas del hombre y no al contrario.

La empresa colombina S.A., en busca de satisfacer a sus clientes nacionales e internacionales con productos de excelente calidad bajo los estándares internacionales de las normas ISO 9001:2008 en calidad Bajo la normatividad técnica de calidad en la gestión pública NTCGP 1000:2009, las normatividad de Buenas Prácticas de Manufactura (BPM decreto 539 de2014) tomado de la resolución 2674 de 2013 la en la elaboración de productos inocuos y bajo los estándares del sistema de gestión ambiental ISO 14001:2004 con la Norma técnica colombina NTC -ISO 14001:2004 entre otros.

Dentro de la variedad de productos se hace referencia a la elaboración de la pastilla de chocolate Chocodisk donde para realizar este proceso una persona debe de alimentar dos máquinas de forma manual, de manera que para alimentar una de las máquinas debe coger los recipientes con producto Chocodisk que se encuentran en una estiba desde el piso transitar con el carro y subir las conchas a una plataforma de 2 metros de altura donde luego el personal sube a la plataforma y luego vierte el producto a la tolva de la máquina cogiendo 5 veces la misma concha de manera que haciendo estas actividades para las dos máquinas el personal carga alrededor de 17 toneladas en un turno de 8 horas. Mientras que para alimentar la tercera máquina esta cuenta con un personal y esta máquina tiene su tolva en la parte inferior a una altura de un metro y al lado se encuentra su producto facilitando la cargada o alimentación de este.

Actualmente para realizar el envase del producto la empresa colombina cuenta con tres máquinas donde dos de ellas se alimentan de forma manual y una por banda transportadora donde en la alimentación manual su proceso ha generado incapacidades, enfermedades laborales, restricciones médicas, accidentes laborales conflicto en el grupo de trabajo, incumplimiento en los indicadores de producción debido a que hay que coger el producto desde piso, subirlo, al carro, transportarlo hasta una plataforma nuevamente, subirlo a la plataforma, luego el personal se desplaza por una escalera diagonal, sube a la plataforma realiza el arrume de los recipientes que subió para posteriormente abastecer el producto en las tolvas de la máquina.

Las trabajos presentados de salud y riesgo nos permiten tener un idea de la importancia del sistema, por ejemplo: (Marín, 2006) clasifica los accidentes de trabajo según el lugar donde ocurren, sucedidos en el lugar o centro de trabajo, yendo o viniendo del trabajo, o según la situación laboral del trabajador; con baja o sin baja, y según la gravedad de la lesión a criterio médico.

De igual forma, es necesario tener en cuenta las causas de los accidentes de trabajo; para (Cortés, 2007), las causas se entienden como las diferentes condiciones o circunstancias materiales o humanas que aparecen en el análisis del mismo. Es posible deducir una clasificación dependiendo de su origen las cuales pueden ser técnicas y humanas.

Una condición insegura, comprende el conjunto de circunstancias o condiciones materiales, mientras que un acto inseguro, comprende el conjunto de actuaciones humanas a las que se conocen también como actos peligrosos, prácticas inseguras o factor humano.

(Muñoz, 2004), define las causas de accidente de trabajo como las disfunciones latentes en el seno de la organización que propician la creación de condiciones y actos inseguros, siendo dichas condiciones y actos simples manifestaciones de deficiencias estructurales $o$ perturbaciones operativas del centro de trabajo. Estas causas son las que permiten explicar porque sucede lo indeseable, materializado en hechos concretos que están posibilitados por previos comportamientos de los operarios. 
Los accidentes de trabajo conllevan unos costos, de acuerdo con (Muñoz, 2004), el concepto económico del accidente de trabajo ha hecho que los países industrializados se le consideren como un costo adicional de producción.

Para (Chicnhilla, 2005), es un elemento que permite medir el impacto de un accidente laboral, lo que constituye la estimación del costo. Todo accidente tiene un costo para el trabajador, el dueño de la empresa y el consumidor, que puede ser judicial, médico o social.

Recursos Humanos en la Empresa. Desde el punto de vista de (Lledo, 2011), se afirma que los recursos humanos tienen un enfoque de aplicación y practica de las actividades más importantes dentro de la organización o empresas siendo la gestión del talento humano un pilar fundamental para el desarrollo exitoso de los procesos, pues al final las personas son los responsables de ejecutar las actividades porque los proyectos no se desarrollan por si solos. La administración de recursos humanos (ARH) es el proceso de contratar, capacitar, evaluar y remunerar a los empleados, así como de atender sus relaciones laborales, salud y seguridad, así como aspectos de justicia (Ramírez, 2007; Dessler, 2009).

Lo planteado anteriormente, lleva a la formulación de la pregunta de investigación: ¿Cómo mejorar el sistema de alimentación en las máquinas envasadoras Multipak y Rovema, que permita la reducción del riesgo ergonómico y los tiempos improductivos de operación?

\section{Esto permitirá:}

- Minimizar la manipulación del producto reduciendo el almacenamiento masivo durante el proceso, garantizando productos inocuos y de excelente calidad.

- Utilizar el elevador de la máquina Robat para mejorar el proceso de alimentación de las máquinas Multipack y Rovema, aprovechando la altura del elevador ya existente.

- Eliminar los tiempos excesivos de paros al inicio del proceso productivo en la alimentación de las máquinas envasadoras para aumentar el volumen de producción.

El área de chocolatería considera evaluar una de sus líneas de producción a fin de implementar un sistema de alimentación de forma tal que el elevador de la tercera máquina envasadora Robat alcance a alimentar las máquinas Multipack y Rovema por este sistema con solo llenar manualmente la tolva de la Robat.

Es de aclarar, que con este sistema se facilita la alimentación de las máquinas eliminando así los esfuerzos ergonómicos inadecuados, maximizando los tiempos productivos, la moral, el bienestar y la salud para el personal operativo.

\section{METODOLOGÍA}

La metodología a utilizar durante la investigación e implementación del proyecto se enfoca en el rediseño y mejora de las actividades de un proceso con la finalidad de hacerlo más eficiente y eficaz logrando que este brinde resultados satisfactorios al que tenía anteriormente y que sus acciones sistemáticas logren ser más estables.

El rediseño de estos procesos incorpora actividades de mejoras permanentes, dado a que en la mejora en sí, se aplica el ciclo PHVA de mejora continua. El ciclo PHVA fue desarrollado por Walter Stewart y aplicado por el doctor Deming a partir de la década de los 50, la mejora continua del sistema en el ciclo PHVA; este es un ciclo dinámico que se desarrolla en todo el sistema de gestión, como al interior de cada proceso sea directivo, fundamental o de soporte. Las etapas que involucra el ciclo PHVA para el rediseño y mejora se compone de cuatro etapas: planear, hacer, verificar, ajustar.

En la etapa de planear, "consiste en estudiar la situación actual, recolectar información y genera un diagnóstico. Dentro de las actividades que se desarrollan en esta etapa se encuentran la definición del proceso, sus insumos, resultados, cliente proveedores. Identificar las expectativas del cliente. Identificar problemas y desarrollar posibles soluciones que también hacen parte de las actividades a desarrollar".

$\checkmark$ En la etapa de hacer, "se inician prácticas, es decir, se desarrolla una prueba piloto plan anteriormente realizado, Esta implementación limitada es un experimento para evaluar una solución propuesta y brindar los datos objetivos". Datos necesarios que apunten a los objetivos planteados para la fiabilidad de los pasos a seguir en el desarrollo de la ejecución de la mejora. 
En la etapa de verificar, "durante esta etapa se busca Diagnosticar si durante el ensayo el plan funciona correctamente, o si se han presentado problemas $\mathrm{u}$ oportunidades adicionales, se procede a nuevas soluciones evaluándose con la necesidad y luego pasar a la etapa hacer". Con la exigencia que se cumpla con los objetivos que se deben de tener hasta la etapa actual, garantizando que en la etapa siguiente no se tengan problemas ya anunciados anteriormente.

$\checkmark$ En la etapa de actuar, "ajustar el plan final, posterior a esté. Se realizan prácticas estableciendo que las mejoras se convierten en normas". Si se cumple con el objetivo planteado en esta etapa estas normas se estandarizaran para que los resultados en las actividades de la mejora se mantengan, de lo contrario se deberá de corregir la anomalía. Hasta que se cumpla el objetivo planificado en esta etapa. Siendo esta última la que acredita el sistema de alimentación a futuro.

\section{Herramientas para análisis de los procesos de investigación}

Un aspecto particularmente importante en la investigación para mejorar los procesos productivos o estándares de producción, es la utilización de herramientas que sean fundamentales a la hora de evaluar alguna actividad o estándar de producción en beneficio de mejores resultados en sus procesos, bien sea de mejorar en la parte administrativa en sus procesos manufactureros o servicios de la organización de (Chiavenato, 2009). A continuación se presentan las técnicas empleadas que son de gran importancia para el desarrollo de la investigación.

Definición de estudio de tiempos. Consiste en establecer estándares de tiempos, para lo cual se han empleado tres medios para determinar las: estimaciones, registros históricos y medición del trabajo. Tanto el método de registro histórico como el de medición de trabajo, dan valores mucho más exactos que el de las estimaciones básicas en juicios o apreciación personal.

En el método de los registros históricos, los estándares de producción se basan en los registros de trabajos semejantes realizados con anterioridad (Caballero A. et al., 2013). Los estándares de tiempo cuidadosamente establecidos posibilitan una mayor producción en la planta, incrementando así la eficiencia del equipo y del personal que lo opera.
Algo muy fundamental para planear el rediseño y las posibles mejoras es conocer los procesos con sus causas, paros y fallas, los cuales interrumpen el flujo de las actividades de sus procesos con el concepto de lo que no se mide no se mejora. Con estos conocimientos y metodología se tienen etapas para el inicio y realizar los respectivos análisis. Como primer paso contar con el liderazgo de la Alta Dirección y Líderes de la Organización que aseguren y ofrezcan los recursos necesarios para la ejecución de la mejora.

\section{Método Rula}

Desarrollado para evaluar la exposición de los trabajadores a factores de riesgo que pueden ocasionar trastornos en los miembros superiores del cuerpo, proporcionando una valoración rápida de las posturas del cuello, tronco, y miembros superior, junto con la actividad muscular y las fuerzas o cargas experimentadas por el trabajador.

Diseño de la mejora: De acuerdo a la tercera maquina Robat que tiene una banda transportadora y supera la altura de las dos máquinas de alimentación manual se realiza una prueba piloto implementando un tipo de tobogán desde la parte superior de la robat y que finalice en las tolvas de la maquina multipack y rovema para que estas sean alimentadas por la banda transportadora. Cayendo el producto por caída libre (Torres et al., 2013).

\section{RESULTADOS}

Para la realización de la Rula, se tienen en cuenta las siguientes normas:

- GTC 45- Identificación de peligros y valoración de riesgos

- Resolución 2400 del 1979

- NTC 5693-1: Manipulación manual parte 1: levantamiento y transporte.

- NTC 5693-2: Manipulación manual parte 2: empujar y halar.

- NTC 5693-3: Manipulación manual parte 3: manipulación e cargas livianas a alta frecuencia.

Resultados Ergonómicos.

Análisis Ergonómico método Rula. Para el análisis de las condiciones ergonómicas no adecuadas se emplea el método rula, De modo que realiza un seguimiento minucioso con sus diversas técnicas que evalúan las posturas y condiciones del puesto 
de trabajo. Para (Cuesta, 2010). Evaluación ergonómica de puestos de trabajo. R.U.L.A (Rapid Upper Limb Assessment), en su traducción al castellano "evaluación rápida de la extremidad superior". Durante los estudios realizados en la implementación del sistema de alimentación se logra la eliminación de actividades que presentan un alto riesgo de accidentalidad y situaciones ergonómicas no adecuadas. En el procedimiento para la ejecución de la mejora del sistema de alimentación es evidente las mejores condiciones en la alimentación de las máquinas beneficiándose en primera instancia el grupo de operarios alimentadores. Siendo el propósito del sistema de alimentación.

La tabla 1 a continuación presenta la evaluación de la rula Multipack y Rovema en el grupo A y B realizada la mejora para el proceso del sistema de alimentación. Lográndose disminuir el nivel de riesgo desde un $50 \%$ hasta un $0 \%$ en las actividades de alimentación. Es importante aclarar que algunas condiciones se modificaron y cambiaron, pero estos análisis no definen eliminación del riesgo en el proceso de alimentación.

Tabla 1. Rula de Evaluación de puestos de trabajo (Fuente: Fisioterapeuta Carolina Gómez Arboleda).

Grupo A : análisis de brazo, antebrazo y muñeca:

\begin{tabular}{|c|c|c|c|}
\hline DESCRIPCIÓ & NIVE & CALIFICACIO & DISMINUCIÓ \\
\hline $\mathrm{N}$ & & $\mathrm{N}$ & $\mathrm{N}$ \\
\hline $\begin{array}{l}\text { Puntuación del } \\
\text { brazo }\end{array}$ & $(1-6)$ & 3 & $50 \%$ \\
\hline $\begin{array}{l}\text { Puntuación del } \\
\text { Antebrazo }\end{array}$ & $(1-3)$ & 2 & $67 \%$ \\
\hline $\begin{array}{l}\text { Puntuación de } \\
\text { la muñeca }\end{array}$ & $(1-4)$ & 2 & $50 \%$ \\
\hline Puntuación & $(1-2)$ & 1 & $50 \%$ \\
\hline $\begin{array}{l}\text { Giro } \\
\text { muñeca }\end{array}$ & & & \\
\hline $\begin{array}{l}\text { Puntuación } \\
\text { Grupo }\end{array}$ & $(0-1)$ & 1 & $100 \%$ \\
\hline $\begin{array}{l}\text { Muscular } \\
\text { (Grupo A) }\end{array}$ & & & \\
\hline $\begin{array}{l}\text { Puntuación de } \\
\text { Carga /fuerza } \\
\text { (Grupo A) }\end{array}$ & $(0-3)$ & 0 & $0 \%$ \\
\hline \multicolumn{4}{|c|}{ Grupo B : análisis de Cuello, Tronco y Piernas: } \\
\hline $\begin{array}{l}\text { DESCRIPCIÓ } \\
\mathrm{N}\end{array}$ & $\begin{array}{l}\text { NIVE } \\
\mathrm{L}\end{array}$ & $\begin{array}{l}\text { CALIFICACIO } \\
\mathrm{N}\end{array}$ & $\begin{array}{l}\text { DISMINUCIÓ } \\
\mathrm{N}\end{array}$ \\
\hline $\begin{array}{l}\text { Puntuación del } \\
\text { Cuello }\end{array}$ & $(1-6)$ & 2 & $33 \%$ \\
\hline $\begin{array}{l}\text { Puntuación del } \\
\text { Tronco }\end{array}$ & $(1-6)$ & 2 & $33 \%$ \\
\hline $\begin{array}{l}\text { Puntuación de } \\
\text { Piernas }\end{array}$ & $(1-2)$ & 1 & $50 \%$ \\
\hline $\begin{array}{l}\text { Puntuación } \\
\text { Grupo } \\
\text { Muscular } \\
\text { (Grupo B) }\end{array}$ & $(0-1)$ & 1 & $100 \%$ \\
\hline Puntuación de & $(0-3)$ & 2 & $67 \%$ \\
\hline
\end{tabular}

Carga /fuerza

(Grupo B)

\section{NIVEL DE RIESGO Y ACTUACIÓN}

DESCRIPCIO NIVE CALIFICACIO DISMINUCIÓ

\begin{tabular}{llll}
$\mathrm{N}$ & $\mathrm{L}$ & $\mathrm{N}$ & $\mathrm{N}$ \\
\hline Puntuación & $(1-7)$ & 5 & $71 \%$
\end{tabular}

final Rula

$\begin{array}{llll}\text { Nivel de } & (1-4) & 2 & 50 \%\end{array}$

Riesgo

Conclusión: La mejora realizada a este puesto le dio mayor confort a la actividad realizada, durante la alimentación de las tolvas.

Para mayor interpretación en los cambios que arroja la rula en la evaluación del nivel de riesgo ergonómico en la Máquina Multipack y Rovema teniendo en cuenta el nivel de riesgo de la Tabla 1 y Tabla 2 se presenta en el gráfico comparativo (Figura 1) el grupo A Sin presentar cambio alguno en la puntuación muscular. Debido a que el nivel de riesgo es de (0-1) mientras que en la carga fuerza su nivel de riesgo es de (0-3) arrojando un porcentaje relevante del 0\%. En la (Figura 2) se tiene el grupo B Igual sin ningún cambio en el análisis muscular, por último en la (Figura 3) se tiene el grafico puntuación final que arroja la rula y el nivel de riesgo disminuyendo este en un $25 \%$.

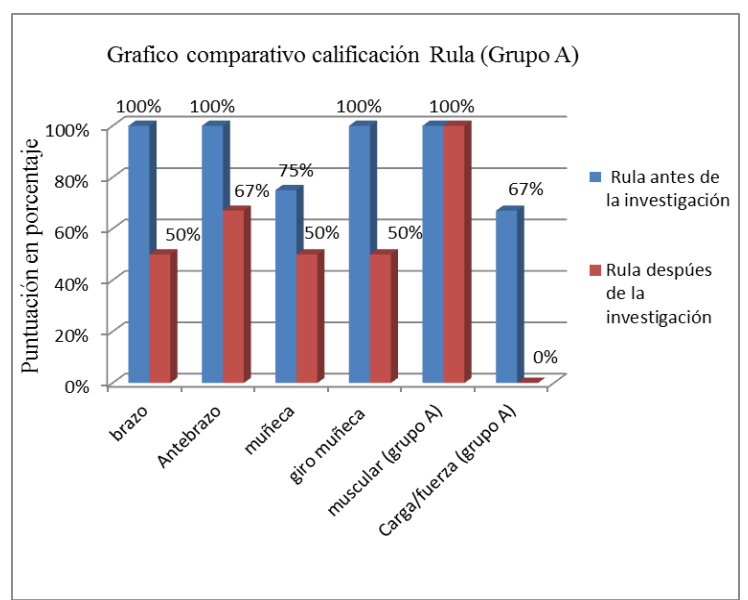

Fig. 1. Resultado evaluación Rula Grupo A.

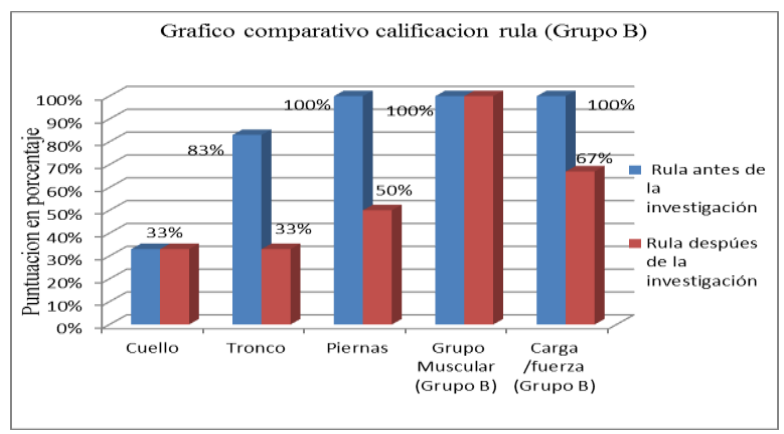

Grafico 2. Resultado evaluación Rula. Grupo B. 


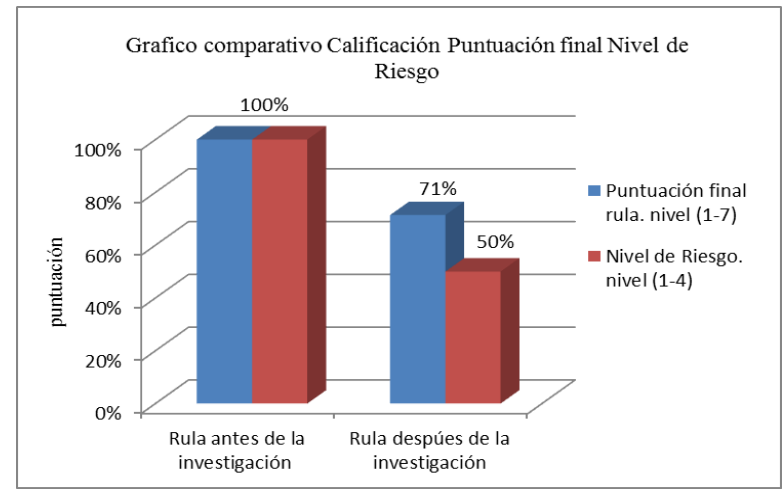

Figura 3. Nivel de Riesgo y Actuación.

Procedimiento en el Sistema de Alimentación. Durante el proceso de elaboración de la pastilla de chocolate Chocodisk de la línea de grajeados, antes de iniciar labores operativas en el proceso de alimentación, la máquinas se debe abastecer o tener un inventario considerado de máximo diez (10) conchas de producto para la puesta en marcha del proceso de envase; Para el proceso de alimentación se tienen dos opciones, la primera es coger el producto desde la estiba y la segunda opción tomar el producto desde el bombo de brillado. Se alimenta la tolva de la Robat hasta llenar y de esta forma se estará alimentando la tolva de la Multipack y Rovema. Sin requerir mayor cantidad de producto inventariado que se exponga a manipulación. Solo se llena la tolva de la robat para iniciar el proceso de envase.

La optimización en los tiempos del proceso de alimentación que se presenta con la propuesta de la mejora permite que el personal operativo tenga más tiempo en la atención de las máquinas Multipack y Rovema para que estas no queden en espera de personal operativo cuando este esté realizando funciones diferentes en la atención a las máquinas envasadoras. Esta es una opción muy favorable para que se cumplan los indicadores estándares de producción en el proceso de alimentación, en donde anteriormente de acuerdo a los estudios de tiempo realizado el tiempo total para alimentar las maquinas Multipack y Rovema en el turno correspondía a 2,55 horas y con la ejecución de la mejora solo será de 0,7 horas. Como se verá en la tabla siguiente:

Tabla 2. Tiempos en Alimentar las Máquinas.

\section{Resultado/}

Producto

Esperado
Beneficiario

\begin{tabular}{|c|c|c|c|}
\hline $\begin{array}{l}\text { Optimizació } \\
\text { n de tiempos } \\
\text { operacionale }\end{array}$ & $\begin{array}{l}\text { Tiempo } \\
\text { para } \\
\text { alimentar }\end{array}$ & $\begin{array}{l}\text { Tiempo } \\
\text { para } \\
\text { alimentar }\end{array}$ & $\begin{array}{l}\text { Personal } \\
\text { operativo, } \\
\text { Producción }\end{array}$ \\
\hline $\mathrm{s}$ en las & Rovema & Multipac & del área de \\
\hline maquinas & $\begin{array}{l}\text { y la } \\
\text { Multipac }\end{array}$ & $\begin{array}{l}\text { k } \\
\text { Rovema }\end{array}$ & chocolatería. \\
\hline Optimizació & $\mathrm{k}$ & y Robat & Departamento \\
\hline $\begin{array}{l}n \quad \text { en los } \\
\text { tiempos de } \\
\text { alimentación }\end{array}$ & $\begin{array}{l}2,55 \\
\text { horas }\end{array}$ & 0,7 horas & $\begin{array}{l}\text { de Control } \\
\text { calidad }\end{array}$ \\
\hline
\end{tabular}

En términos de porcentaje se disminuye un $78 \%$ del tiempo en alimentar las máquinas Multipack y Rovema para luego alimentar la Multipack y Rovema y Robat en solo un $22 \%$ del tiempo que se está empleando.

\section{Optimización de los tiempos Máquinas Envasadoras.}

De acuerdo a los estudios planteados para la mejora en el sistema de alimentación, se eliminan las actividades y posturas ergonómicas no adecuadas del proceso de alimentación. Siendo así que los tiempos empleados en las actividades eliminadas, durante el turno y al inicio de turno, ahora son utilizados en la parte operativa de la máquina con la finalidad de lograr un alcance más eficiente en los inicios operacionales para el funcionamiento de las máquinas y cumplir con los estándares programados de producción del área de chocolatería de la que se beneficia toda la cadena de producción hasta ventas (Tabla 3 ).

Tabla 3. Optimización de los tiempos de las máquinas envasadoras.

\begin{tabular}{|c|c|c|}
\hline $\begin{array}{l}\text { Resultado/ } \\
\text { Producto } \\
\text { Esperado }\end{array}$ & $\begin{array}{l}\text { Cumplimiento } \\
\text { productividad }\end{array}$ & ario \\
\hline $\begin{array}{l}\text { Aumento } \\
\text { Productividad } \\
\text { neta por turno }\end{array}$ & $\begin{array}{l}\text { De acuerdo a la optimización } \\
\text { de los tiempos operacionales } \\
\text { se estima un tiempo de } \\
\text { recuperación entre } 40 \text { y } 45 \\
\text { minutos en las } 2 \text { máquinas por } \\
\text { turno. }\end{array}$ & $\begin{array}{l}\text { El área de } \\
\text { Producción, } \\
\text { planeación, } \\
\text { mercadeo, } \\
\text { ventas. }\end{array}$ \\
\hline
\end{tabular}

\section{Ahorro de inversiones de IDP (Ingeniería Desarrollo de Proyectos).}

El departamento del I.D.P (Ingeniería Desarrollo de Proyectos) en la empresa Colombina S.A., es el encargado de la cotización y la compra de equipos y máquinas para el desarrollo y beneficio de la organización; el I.D.P realiza la compra, siempre y cuando el área de producción que solicita el equipo tenga disponible los recursos necesarios para el proceso de compra a lo que está requiriendo; no 
obstante, es necesario realizar la solicitud a gerencia para llegar a acuerdos con el I.D.P.

Teniendo en cuenta la necesidad de producción se presenta la propuesta de mejora del sistema de alimentación mucho más práctico, económico y con excelentes resultados, mejora que cuantitativa y cualitativamente permite que el proceso en el área de grajeados sea más eficaz y eficiente, evitando el gasto en la inversión de un elevador en (Z) y poder utilizar esos recursos en otras áreas que lo estén requiriendo (Tabla 4).

Tabla 4. Ahorro de Inversiones de I.D.P (fuente: Registros Empresa Colombina S.A).

\begin{tabular}{|c|c|c|c|}
\hline $\begin{array}{l}\text { Resultado/ } \\
\text { Producto } \\
\text { Esperado }\end{array}$ & $\begin{array}{l}\text { Cumplimiento } \\
\text { productividad }\end{array}$ & en & $\begin{array}{l}\text { Beneficiari } \\
\text { o }\end{array}$ \\
\hline \multirow{2}{*}{$\begin{array}{l}\text { Ahorro de la } \\
\text { compra de } \\
\text { dos } \\
\text { transportador } \\
\text { es elevador } \\
\text { de producto }\end{array}$} & $\begin{array}{l}\text { Costo } \\
\text { Transportad } \\
\text { or } \\
\text { Nuevo }\end{array}$ & $\begin{array}{l}\text { Costo } \\
\text { Implementaci } \\
\text { ón } \\
\text { De la mejora }\end{array}$ & \multirow{2}{*}{$\begin{array}{l}\text { Departamen } \\
\text { to de } \\
\text { proyectos, } \\
\text { Finanzas de } \\
\text { la } \\
\text { organizació } \\
\text { n. }\end{array}$} \\
\hline & $\begin{array}{l} \\
80.000 .000\end{array}$ & $\$ 4.909 .242$ & \\
\hline
\end{tabular}

El departamento de Ingeniería y Desarrollo de Proyecto (IDP) de acuerdo a las cotizaciones realizadas para la compra de un elevador en $(Z)$ y con el conocimiento de las dos máquinas Multipack y Rovema que no cuentan con un elevador que transporte el producto hasta sus tolvas, cuando toda máquina envasadora en la empresa colombina tiene su propio elevador, ve la necesidad de tomar acciones inmediatas analizando los estudios realizados en la mejora donde se percibe el ahorro de un $93.8 \%$ en la inversión de otro elevador en (Z) (ver Figura 4).

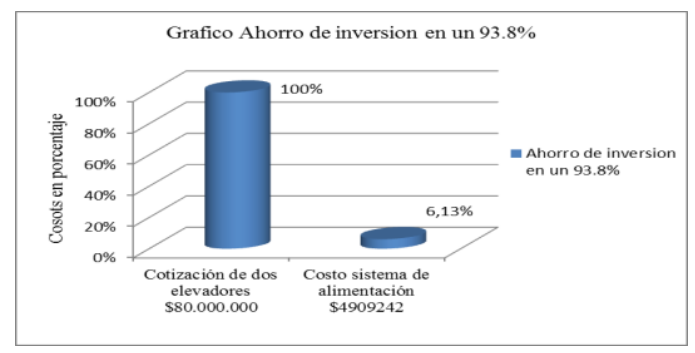

Figura 4. Ahorro del $93.8 \%$ en la Inversión.

\section{CONCLUSIONES}

En la presente investigación se abordaron temas de estudio dentro de los que se destacan los métodos y técnicas de gran importancia para el desarrollo del sistema de alimentación en una empresa manufacturera, lográndose la ejecución de la mejora en las máquinas Envasadoras, beneficiándose de esta manera el recurso humano perteneciente al área de producción, la línea de envase y la organización lográndose los objetivos planteados.

La alimentación de las máquinas envasadoras Multipack y Rovema deja de ser así un sistema de alimentación manual eliminando las tareas que se realizan para alimentar las máquinas envasadoras, realizándose la alimentación por medio de un elevador y luego por el sistema de alimentación implementado, alimentándose simultáneamente las tolvas de las máquinas Robat Multipack y Rovema.

La mejora presenta un mejor confort en la línea de envase de forma que él personal trabaja a gusto, con moral en sus tareas de alimentación, debido a que no se evidencia ningún caso de peligro de accidentalidad o enfermedades laborales para el trabajador.

El proceso en la línea de envase es más eficiente debido a que en el reporte de producción del turno. No se realiza paro relevante por preparación de equipo, De donde para iniciar el proceso de envase en las tres máquinas es solo necesario llenar cierta cantidad con producto la tolva inferior de la Robat Se tiene menos producto inventariado para envasar, resultando ser factible para el cumplimiento en las técnicas de $5 \mathrm{~s}$ y la inocuidad del producto. Garantizando la calidad en el proceso de la pastilla Chocodisk y satisfacer el cliente interno y externo. Concluida la mejora se recomienda a la empresa: Velar que se programe por parte del área de mantenimiento predictivos, preventivos y correctivos con la finalidad de verificar el estado y funcionamiento adecuado del sistema de alimentación, de igual manera en el área de producción se requiere que al sistema de alimentación le realicen aseos semanales o mensuales de acuerdo a la programación de la línea de envase. Logrando así, cumplir con las técnicas de $5 \mathrm{~s}$ y garantizar la inocuidad del producto pastilla de chocolate Chocodisk.

\section{REFERENCIAS}

Caballero Amaury, Velasco Gabriel, Pardo García A. (2013). Differentiations of objects in diffuse databases. Revista colombiana de 
tecnologías de Avanzada. 2 (22). Pág. 131 137.

Cuesta, A. (2012). Evaluación Ergonómica de los Puestos de Trabajo "Rula (RAPID UPPER LIMB ASSESSMENT)” Editorial paraninfo.

Chiavenato, I. (2006). Administración de recursos humanos: fundamentos básicos. (5a. Edición). Brasil: Sao Paulo Atlas.

Chiavenato, I. (2009). Gestión del Talento Humano. (3a. Edición). México: Mc Graw Hill.

Chinchilla, R. (2005). Salud y seguridad en el trabajo. (2a ed.) Costa Rica: EUNED.

Cortés, J. (2007). Seguridad e Higiene del trabajo: Técnicas de prevención de riesgos laborales. (9a. Edición). Madrid: editorial Tebar S. L.

DECRETO 539, (2014). Por el cual se expide el reglamento técnico sobre los requisitos sanitarios que deben cumplir los importadores y exportadores de alimentos. Recuperado de: http://www.alcaldiabogota.gov.co/sisjur/norm as/Norma1.jsp?i=57016\#21

Dessler, (2009). La Administración de los Recursos Humanos. Decimoprimera Edición PEARSON EDUCACIÓN, México, 2009.

Editada por el Instituto Colombino de Normas técnica y Certificación (INCONTEC) Medio ambiente. Gestión Ambiental. NTC -ISO 14001, tomado de: http://www.bogotaturismo.gov.co/sites/intran et.bogotaturismo.gov.co/files/NTC\%20ISO14 001\%20DE\%202004.pdf

Instituto Colombiano de Normas Técnicas y Certificación. Sistemas de Gestión de la Calidad. Requisitos. NTC-ISO 9001. Bogotá D.C.

https://www.invima.gov.co/buscador.html?se archword=decreto\%20ISO\%209001:2008.\&s earchphrase $=$ all

Lledo, P. (2011). La Gestión de Talento Humano. http://desarrolloclima.blogspot.com.co/2013/0 9/talento-humano.html

Marín, F. (2006). Seguridad industrial manual para la formación de ingenieros. Madrid: editorial Dykinson.

Muñoz, J. (2004). La gestión integrada: calidad, seguridad y medio ambiente. España: SERFOREM, S.L

NTC 5693-1: Manipulación manual parte 1: levantamiento y transporte.

NTC 5693-2: Manipulación manual parte 2: empujar y halar

NTC 5693-3: Manipulación manual parte 3: manipulación e cargas livianas a alta frecuencia

Ramírez, C. (2007). Seguridad industrial un enfoque integral. (3a. Edición). México: Limusa.

R.U.L.A (Rapid Upper Limb Assessment), en su traducción al castellano "evaluación rápida de la extremidad superior".

Torres Clayton José, Archila John Faber, Tronco Mário Luiz, Becker Marcelo, Viera Porto Arthur José, Tiberti Alexander José. (2013). Estudio cinemático de una plataforma robótica para agricultura. Revista colombiana de tecnologías de Avanzada. 2 (22). Pág. 131 $-137$.

https://books.google.com.co/books?id=v5kFfWOU $\mathrm{h} 5 \mathrm{oC} \&$ printsec $=$ frontcover \&dq=inauthor: $\% 2$ 2Sabina+Asensio-Cuesta\%22\&hl=es419\&sa=X\&ved=0ahUKEwjluIGnydvOAhW JMx4KHT_3B6gQuwUIHzAA\#v=onepage \& $\mathrm{q} \& \mathrm{f}=$ false 